\title{
Problem-based Learning in Virtual Worlds: Two Case Studies in User Interface Design
}

\author{
Spyros Vosinakis, Panayiotis Koutsabasis, Panagiotis Zaharias, \\ Marios Belk
}

\begin{abstract}
Problem-based Learning (PBL) is an educational approach that is based on student collaboration and self-directed learning. In PBL, students learn by addressing illdefined and open-ended problems reflecting on their experiences. This paper aims to investigate the suitability of Virtual Worlds (VWs) as a platform for hosting PBL activities and to report on their strengths and difficulties in terms of usability, collaboration support and learning effectiveness. We have set up a VW on the basis of open source software, developed a number of in-world supporting tools, and ran two PBL activities in the area of User Interface Design. Students were asked to collaboratively design and present the user interface of applications in various problem areas and platforms. Each group collected resources, presented and argued about concepts, and built together an interactive user interface prototype with explanatory annotations. The final prototypes were presented to the class. The learning activities have been evaluated and the results have revealed several strong points of VWs that validate their potential for PBL activities, but also indicated a number of problems to be tackled.
\end{abstract}

Key Words: Virtual Worlds, Virtual Environments, Education, Problem-based learning, User Interface Design.

$* * * * *$

\section{Introduction}

Problem-based learning as a learning and teaching practice has been widely adopted during the last 20 years in both traditional and online educational settings. ${ }^{1}$ In PBL students learn by addressing ill-defined and open-ended real-life problems collaboratively, usually without prior knowledge about the problem domain. During the process students identify their knowledge deficiencies, decide what they need to learn, propose solutions, evaluate them and reflect on their experiences, thus developing problem-solving strategies and building domain knowledge in a self-directed manner. ${ }^{2}$ This approach has several advantages, as students are actively gaining transferable skills by investigating, explaining and resolving meaningful problems. Group participation in problem-solving activities as well as the fact that they are working on real-life problems rather than simplified ones, are highly motivating factors for them. Studies have shown that students following a PBL approach have developed more effective problem-solving and self-directed learning skills without having significantly lower performance in 
understanding facts and concepts, compared to students following traditional approaches. $^{3}$

Collaboration and interaction are some of the critical factors for PBL success in both traditional and online learning settings. ${ }^{4,5,6}$ Therefore, it is not surprising that PBL has been characterized as one of the most appropriate learning methods in virtual worlds. ${ }^{7}$ VWs provide great opportunities for both synchronous and asynchronous learning and are highly collaborative, interactive and persistent 3D environments. Instructors and educators can prepare learning materials as in-world tutorials and develop interactive learning scenarios, and students/learners are invited to interact with the world and become active participants in these scenarios. However, the majority of institutions that currently use VWs as part of their curricula simply employ them for resource sharing and conferencing, and the common activities that take place within the environment are text or voice-based communication, document storage and exchange, group discussions and presentations. $^{8,9,10}$ These approaches do not exploit the powerful affordances of VW in presenting real-time simulations of custom environments, in which users can actively participate in an experiential and constructivist manner.

Up to date there are only a few approaches attempting to realize PBL and other constructivist learning methods in VWs. ${ }^{11,12,13}$ This is to some extent reasonable since that VWs are a new medium that is currently being explored in many dimensions with respect to their affordances for learning. Related studies pose problems to students that need to be pursued in the VW. However, in many of these cases important PBL principles are not followed mainly because authentic and ill-defined problems are not presented to students, who in turn are not engaged in self-directed learning and deep critical thinking, but in mere problem solving. In addition, much of the relevant research on PBL is still conceptual and information about detailed evaluation with regard to specific methods and practices is lacking. We argue that VWs should be explored for setting up novel educational interventions that support and visualize evolving in-world activities with the presence and participation of people who construct and manipulate 3D objects and tools.

The aim of our work is to explore meaningful ways for the facilitation of collaborative PBL activities in VWs. We present the design and evaluation of two PBL interventions in the area of user interface design. Our goal was to engage students in PBL activities through their collaborative design, experimentation and evaluation of user interface prototypes. We designed an educational environment on top of an existing VW platform, built a number of supporting tools for collaboration and prototyping, and facilitated a set of learning activities over two studies. The first intervention was a laboratory study that occurred as an additional lecture in undergraduate students of product design engineering, and lasted for a single day. For this study we performed a detailed evaluation of the activities combining various methods for data collection and analysis in order to explore 
dimensions of collaboration, learning and usability. Following the first study, we used the same environment for a field study that was carried out as part of the laboratory course of HCI (Human-Computer Interaction) at a computer science department and lasted two months. In this study we put much emphasis on the evaluation of collaborative learning activities according to the PBL philosophy and were able to obtain qualitative results about the learners' experience with the VW. Our evaluation results and experiences out of these studies reveal several strengths and weaknesses of VWs as potential PBL platforms.

\section{Virtual Worlds as Constructivist Learning Platforms}

Virtual Worlds have a number of important characteristics that can facilitate educational activities. First of all, they can be used to support distant and asynchronous collaboration of learning communities. They offer various tools for communication in real-time, such as voice and text chat, avatar co-presence, gestures, etc, and they also have the means for offline collaboration, such as creating and maintaining resource collections organized using various spatial metaphors, commenting, placing annotations, sending offline messages, etc. Although traditional CSCW (Computer-Supported Collaborative Work) tools may be considerably more effective in some of the aforementioned tasks, VWs have the advantage that they afford all these features in an integrated environment that allows for mutual awareness. This characteristic is very important for the socialization and co-operation of the learning community. ${ }^{14} \mathrm{VWs}$ compared to other learning tools have the unique properties of presence and autonomy. They may generate rich experiential learning environments, through which learners find themselves in realistic or imaginary spaces, perform experiments, observe the results and formulate theories. Given that imitation of reality is not necessary, a virtual learning environment may use custom visual metaphors and rules for the behavior of its objects to present complex abstract concepts in novel ways. ${ }^{15}$ Instructors and learners may also be able to create their own interactive forms to communicate their ideas and concepts in a much more vivid way compared to textual and graphical descriptions. Finally, VWs are an engaging medium that may attract younger learners in a variety of ways.

Based on the aforementioned characteristics, VWs seem to be ideal candidates as constructivist learning environments, because:

- The sense of presence that users feel when immersed in a VW allows them to perceive it as a space they belong to, rather than a digital environment they are interacting with, e.g. a Web site.

- The characteristic of persistence found in VWs lets users reform the space and construct their own meaningful structures.

- The embodiment of users as avatars in the VW allows them to interact with others in richer ways, e.g. using nonverbal forms of communication, compared to other means of mediated interaction, e.g. chat, forums, etc. 
- The expressiveness of animated interactive 3D graphics can be used to present abstract or complex concepts that are difficult to comprehend in a textual form using metaphors, and help learners to interpret the environments or even construct their own interpretations and communicate them to their peers.

- The real-time simulation and $3 D$ interaction capabilities of VWs can be exploited to implement the appropriate tools and devices for experiential learning and problem solving.

There is not much research on how PBL and other constructivist learning methods can be realized in VWs. Girvan and Savage proposed Communal Constructivism as a potentially appropriate pedagogy for use in Second Life. ${ }^{16}$ According to Communal Constructivism learners try to build knowledge not only with a focus on themselves but for other future learners as well. Some distinctive features of this pedagogy are the interaction with the environment to construct knowledge, the active collaboration of learners and the transfer of knowledge between groups. Bignell and Parson claim that one of the most appropriate learning methods in virtual worlds, especially in Second Life, is Problem-based Learning. ${ }^{17}$ They support that instructors and educators can prepare learning materials as immersive tutorials and develop interactive learning scenarios, and students/learners can be invited to interact with the world and become active participants in these scenarios, usually in small groups.

A number of case studies have been presented in the last few years that involved PBL activities in VWs. Brown et al demonstrated the suitability of SL for problem-based learning through the mapping of learning activities on to PBL goals, as they have been stated in the framework of Hmelo-Silver. ${ }^{18}$ The problems posed to the students were to create short video clips from their activities in SL using the technique of machinima, i.e. the generation of cinematic video by capturing in real-time the rendered screen of VWs. According to the findings it is supported that development of wider transferable skills can be realized effectively through virtual worlds such as SL. In another related study, Good et al reported findings from a case study with a 'strong' PBL approach where students were tasked to create learning experiences within SL for external clients. ${ }^{19}$ The emphasis was mainly placed on the process of how students formed groups and created the interactive learning experiences by using the affordances of SL. It was found that SL can contribute to PBL as a pedagogical approach in several ways such as supporting the roles of tutors and students, facilitating their relationships, enhancing students' motivation and ownership of the project, as well as easing the assessment activities by the tutors.

Omale et al sought to discover how unique attributes of $\mathrm{VW}$, such as the avatars, the 3D space and the bubble dialogue boxes are being used for PBL activities. ${ }^{20}$ They present a case study of an online PBL environment in which participants were asked to select appropriate learning theories and instructional 
strategies for a science education program. The evaluation results suggest that the VW had a positive impact on the learners' social presence, but learning was not enhanced; rather, they were distracted by the technology. Also, the evaluation results showed that the VW attributes promoted negotiation, clarification and brainstorming among participants, but had less influence on organizational issues.

Vrellis et al used Second Life (SL) to implement a collaborative problem-based learning activity following a constructivist approach. ${ }^{21}$ The VW presented a simple physics experiment (calculating the direction of a bullet in a shooting task) and pairs of students had to collaboratively propose a solution to the problem using a calculator, two rulers and a shared whiteboard. The empirical results highlighted several advantages of VWs for constructivist learning, such as the persistence of the environment, the in-world object manipulation and the use of learning tools.

Notwithstanding the value of these approaches for investigating the appropriateness of VWs as constructivist learning environments, these works do not fully conform to important PBL principles mainly because they do not pose authentic, ill-defined problems to students who in turn are not engaged in selfdirected learning and deep critical thinking. In some cases the problem domain was related to activities that are inherently supported by VWs, e.g. machinima production, and in other cases the 3D environment has been mainly used for group discussions and brainstorming. In addition, constructivist activities such as the use of tools to collaboratively experiment with various problem solutions have not been included.

\section{A Virtual World for User-Interface Design}

The authors have set up a VW as a platform to host PBL activities in User Interface Design. The implementation has been based entirely on open source software. The world server was installed in a standalone PC using the OpenSimulator platform ${ }^{22}$, and the FreeSwitch server $^{23}$ has been set up and connected to the environment to provide voice communication support.

The implementation of PBL activities in VWs poses strong requirements on the environment's side concerning their affordances to fulfill the communication and collaboration needs of remote students and teachers. A necessary prerequisite to detect such requirements is to identify the common student tasks during a session and to discover the collaboration needs for each task. For our study in the area of user interface design we have identified the following tasks in group-based PBL activities:

- In the early stages, students discuss about the problem, write down facts and reveal aspects for which further knowledge may be required.

- Then, they assign roles to group members, search for and share resources, and formulate, present and explain their ideas.

- Finally, they collaboratively assemble a final solution, refine it and present it to the class to be further evaluated. 
The platform we have employed for our study provided inherent support only for part of these tasks. We have therefore implemented a number of additional tools that were available to students during the study in order to overcome these obstacles and to enhance the collaboration affordances of the environment. The implemented tools were:

- Resource: an object that links to external web resources. It can be used by the teacher(s) in order to provide some initial resources to the students (guidelines, design patterns, templates, etc.) to aid them during their tasks, and by the student groups in order to share and organize the resources they found in their self-directed learning activities.

- Comment Recorder: a tool to record and playback user messages. It can be used to take notes from conversations during the early collaboration stages and also as a tool to record viewer comments during the final evaluation stage.

- Annotation: an object that contains a written message. Annotations can be used for the asynchronous collaboration between group members (e.g. in the form of comments, notes about things to be done, role descriptions, etc.) or they may be attached to the user interface prototype as further notes or explanations of design choices.

- Interface Element: an object with scripted behavior that can be used as a user interface component in the working prototype. Students can combine and configure copies of the "Interface Element" object in order to design buttons, windows and image containers during the final stages of the learning activity and collaboratively construct an interactive user interface prototype.

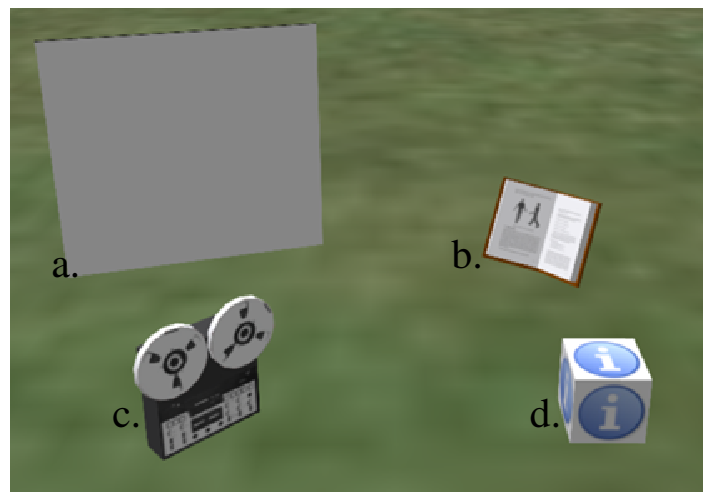

Image 1 - The supporting tools of the environment. a. Interface Element, b. Resource, c. Comment Recorder, d. Annotation 
The aforementioned objects were provided to each student on initialization and they could insert multiple copies of them inside the environment. Image 1 presents a screenshot of the four tools.

\section{User Studies}

\section{A. Laboratory Study}

The first study was an exploratory laboratory experiment that simulated the use of VWs for user interface design. The participants were ten students, who had considerable experience in user interface design, since that they had all attended the courses of: human-computer interaction, interaction design and multimedia design. The participants were allocated in three equivalent groups in terms of their experience in VWs and their user interface design skills and they communicated with an audio link and text chat, thus simulating a remote collaborative work situation.

The problem-based learning activity was given to the participants in the following statement of a 'design brief': "Design the user interface of a multimedia kiosk system for browsing available rooms to let in the island of Syros. The intended users are tourists (Greeks and foreigners), who can access the system from the harbour of Syros. You should take into account usability guidelines for multimedia presentations and information seeking. You should design the 5-7 most basic screens of the system, in wireframes". In addition, the participants were presented with an abstract work plan that included several tasks that they could choose to follow with indicative times for completion.

The learning goals of this intervention were: a) to discover the usability and accessibility requirements of touch screen interfaces, b) to understand the differences in the design of such interfaces compared to other, more conventional cases, and c) to apply this knowledge in a specific practical context.

We have constructed a mixed (qualitative \& quantitative) method for interaction analysis of problem-based CSCL in VWs. More specifically, we used the following methods:

- Automated monitoring of student behaviour: this was achieved by video capturing of the activity within the VW, logfile analysis with respect to the use of the tools, and observation of the state of the world during and after the exercise.

- Dialogue analysis: voice chat was recorded for most of the exercise and an analysis of utterances was performed. We followed the taxonomy of Fussell et $a{ }^{24}{ }^{24}$ who classify utterances in one of the following content categories: Procedure, task status, reference, internal state and acknowledgement. 
- Students' self-reporting: we used a questionnaire that investigated several aspects of the problem-based CSCL experience, as well as follow-up discussion.

- Tutors' evaluation of the outcome: this was performed during the activity and also after the experiment taking into account all data gathered.

The method for interaction analysis explores the dimensions: 'task performance', 'group functioning', 'social support', and 'learning performance and outcome'. The first three dimensions are those proposed by Daradumis et al, ${ }^{25}$ while the fourth dimension was added to investigate issues of particular PBL process.

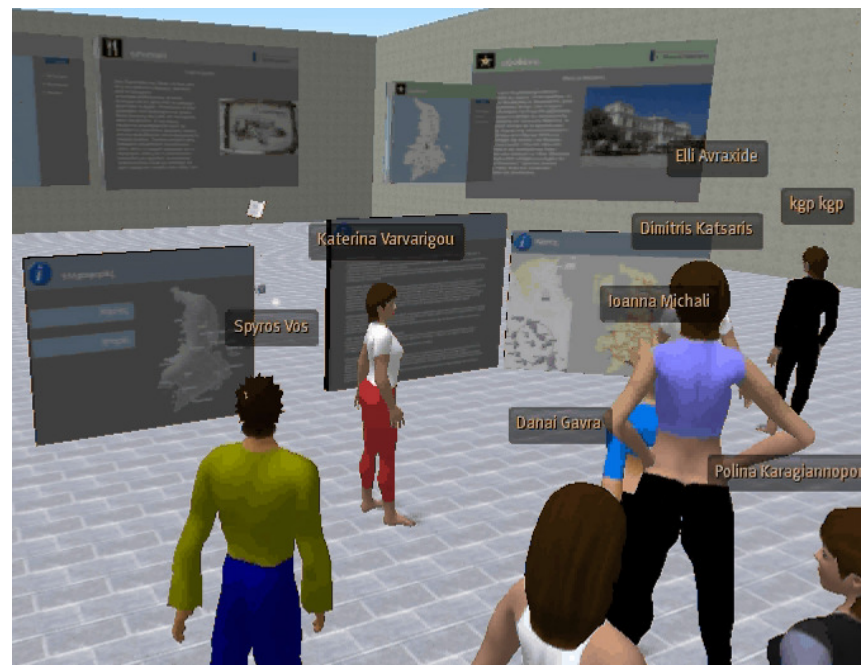

Image 2 - Group presentation inside the VW

The learning intervention lasted for a total time of 6.5 hours. The first 2 hours were devoted to the tutorial about the use of the VW. Then, a total of 3.5 hours were devoted to the activity of user interface design, presentations (Image 2) and follow-up; a total of 1 hour was allocated to the breaks. Participants were asked how much time they would need to carry out the user interface task in a 'face to face' situation and deliver at the same quality: some of them answered about the same time (3.5 hours), others said about an hour less. This is a quite interesting result considering other time consuming activities in face to face situations like for example time and space arrangements.

All teams made use of the tools provided in the environment to document on the design process (Table 1). 
Table 1 - What tools of the VW contributed to the development of your knowledge about the problem? (Bad $122 \quad 3 \quad 4 \quad 5 \quad 6 \quad 7 \quad 8 \quad 9 \quad 10$ Excellent).

\begin{tabular}{lllll} 
& Average & Median & Mode & St.Dev. \\
Resources & 5,5 & 7 & 7 & 2,9 \\
Annotations & 6 & 6 & 6 & 1,6 \\
Comment listener & 4,1 & 5,5 & 0 & 3,7 \\
Interactive objects & 6,7 & 7 & 7 & 1,4 \\
Chat (text) & 7,5 & 8 & 9 & 1,9 \\
Voice Chat & 9 & 10 & 10 & 0,5 \\
\hline
\end{tabular}

With respect to the problem-solving capabilities demonstrated and related actions taken, we observed that students devoted a large portion of their available time to discuss about the understanding of the design problem. These were intertwined with intervals of self-directed learning, which occurred either from 'assignments' or 'requests' by other team mates (e.g. "will you find photos and content about hotels?") or from individual initiative (e.g. "I can find some text to write about Syros history"). This was also identified by the dialogue analysis (Image 3): most of discussion was about the procedure and task coordination $(38.7 \%)$ and acknowledgements $(24.0 \%)$, while less time was devoted to discuss about the task status $(6.2 \%)$ and to refer to virtual objects and tools (11.0\%).

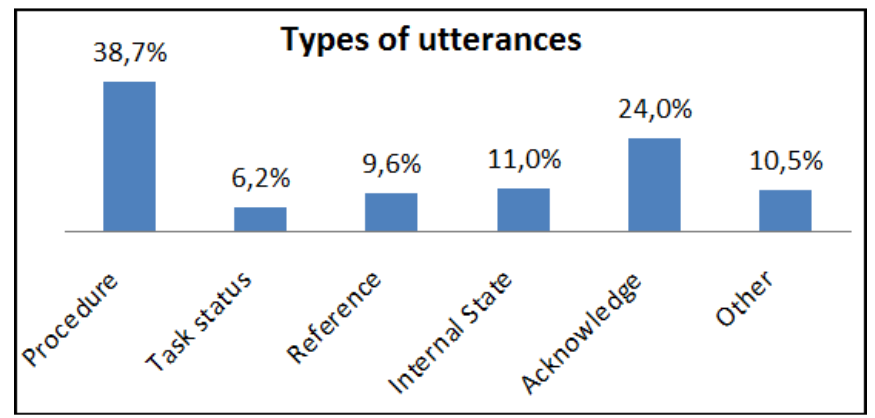

Image 3 - Types of utterances.

Self-evaluation of individual and group performance was quite similar for all participants. The average self-rating of their individual performance regarding the use of the system was: 7 (1: Bad - 10: Excellent) (st.dev.: 1.1). That was pretty much their rating about their team's performance, i.e.: an average of: 7.1 (st.dev.: 1.3). Their responses varied more, when they were asked about their performance with respect to the task of user interface design: they rated their individual performance with an average of 6 (st.dev.: 1.8) and their team's performance with an average of 5.9 (st.dev.: 1.9). Given that we closely observed the process, we 
consider these as rather misbalanced self-assessments: in fact, students faced many difficulties in using the VW, and the fact that they finally achieved to make use of the tools encouraged them to rate their performance rather highly than appropriate. On the other hand, the final outcome of the process was interesting from many aspects. Students have underestimated their performance in this respect because they needed more time for improvements.

With regard to active participation, we observed (mainly from dialogue analysis and self-reporting) that all participants were actively involved in the collaboration and conversations, especially in the first phases of the collaborative activity. All teams exhibited active interaction skills with respect to monitoring the progress of group work; this was evident especially from dialogue analysis: a rather large number of utterances were questions about how to proceed with the activity and specific tasks $(14.1 \%)$, while there were also a large number of acknowledgements (24.0\%) of group work. Each team used a different style of coordination of the work. All participants reported that the result of their work was a collaborative product and that the environment contributed to their collaboration.

Students performed very well with respect to social support: they were all highly motivated and acquainted to each other. More specifically, they rated their commitment towards the achievement of their goal at an average of 7.5 (1: Poor 10: Excellent), and the main reason for this rating not being higher was that some of the participants got carried away out of their curiosity to explore the VW! When they had to wait for other team mates, they kept exploring the world in a playful manner.

Regarding conflict resolution, we identified that there were several disagreements during the learning task about aspects of the design. However, these were openly expressed, discussed and quickly resolved. This is a positive finding: the collaborating participants in the VW seem to be encouraged to individually contribute to group work as well as to constructively resolve conflicts that may arise.

Regarding the learning performance and outcomes, the main result was that all three teams achieved the goal of the exercise (i.e. to provide the design of the user interface of an information kiosk), at a fairly satisfactory level. All teams demonstrated interesting designs that took related guidelines and content into account. However they all reported that they would need more time to elaborate their design solutions. The participants reported that they gradually developed their knowledge about the activity at hand to a considerable extent (an average of $6 ; 1$ : Bad - 10: Excellent; st. dev.: 1.2). They also reported that they devoted about half of the time in self-directed learning: an average of 4.4 (1: None - 10: All; st.dev.:2.3). Also, when asked in which situations they best contributed to the team as with respect to whether they followed the agreed plan, they admitted that their contributions were more suitable when they stick to the plan (an average of $7.2 ; 1$ : Alone - 10: "I stick to the plan"; st. dev.:1.9). 
Regarding the issue of developing problem-solving skills, students first reported on a number of problems faced: most students reported difficulties in using the VW, and a few found it difficult to document their design choices and their opinions with some of the tools provided. Then they reported on their ability to overcome these, an average of 5.2 (1: Bad - 10: Excellent; std. dev.: 2.4). The main reason for not performing better in this respect was that they had limited experience with previous use of VWs. However, we note that the final outcome of the activity, i.e. the user interface design was quite satisfactory for all teams.

The main positive aspects of the experience were identified as follows: (a) "Shared space": Participants felt engaged with the shared space and motivated to work towards their common goal. (b) "Persistence": Also, they highlighted that they could $\log$ off or postpone some of their activities in the world (especially when they performed self-directed learning) and that seeing the world as they had left it was extremely convenient to mentally focus fast on their task. (c) "The VW promotes problem-based collaboration": It was fairly easy to discuss about the task since that they had all material uploaded on the shared space, to compare design ideas and comment on others' work. (d) "Fun, engaging and immersive". Despite the long time in the lab, all participants were kept occupied in the VW (e) "Awareness of others' work, activity and progress", mainly as a motivating factor to one's own work.

The main problems identified are as follows: (a) "Focus on the VW environment, not on the task": some users' attention was for long on the difficulties of using the environment. (b) "Hard to perform organizational tasks": the teams did not manage to keep track of their decisions. (d) "The roles of participants were not mapped to their appearance" (e) "More 2D functions": e.g. the possibility to embed applications from their desktop environment to the VW. (f) "Familiarity with the environment": this was perhaps the most important constraint for this study: none was proficient with the use of a VW, despite some had limited expertise. They felt that if they were more familiar, the final result would be much better.

\section{B. Field Study}

The second study was based on the rationale of the first study but it was more like a field study rather than a laboratory study. Similarly the main purpose was to employ a VW environment for a problem-based learning task: designing user interfaces. The participants were forty students at the Computer Science Department at University of Cyprus, who were following the course in humancomputer interaction in the fourth year of studies. 57\% of the participants were male and $43 \%$ were female and their age varied from 20 to 24 .

The main pedagogical goal of this experiment was:

a) to design an interface according to multimedia and usability design guidelines, 
b) to understand the differences and particularities of the design of such interfaces within VWs as compared to the design in a more conventional manner such as using a visual editor and

c) to apply this knowledge in a specific practical context.

The course took place three times weekly, twice for 75 min theoretical lectures every Tuesday and Friday, and once for a 120 min hands-on lab every Wednesday. The course content was primarily concentrated around principles of interaction design, usability and user experience, including key components of HCI such as requirements specification, task analysis, system design, prototype implementation, research methodologies, usability evaluation methods and techniques with an emphasis on heuristic evaluation and usability testing (see Table 1). The teaching team comprised of the authors themselves, who also provided technical support and facilitated the collaboration and learning activities during the whole intervention.

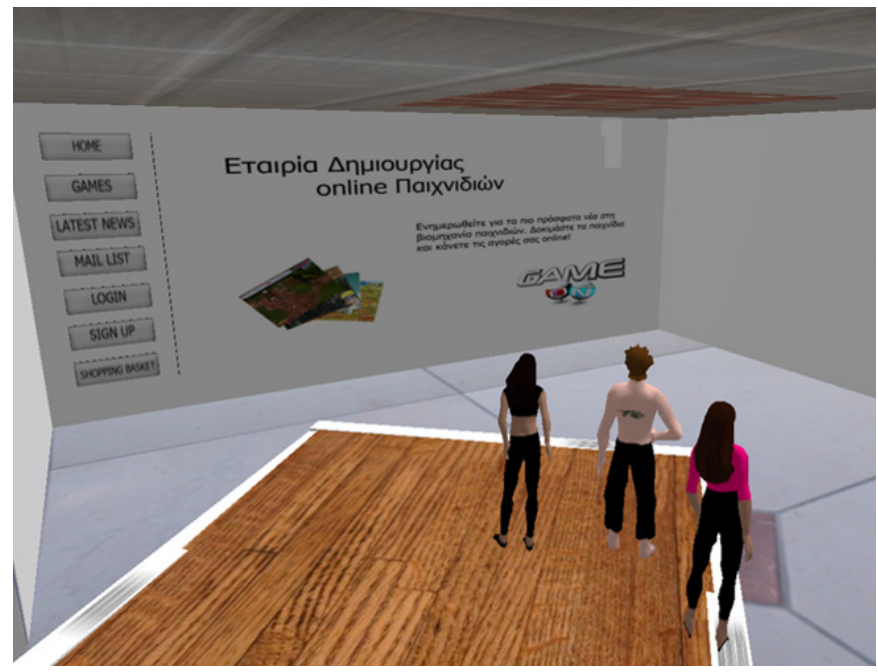

Image 4 - A user interface prototype designed by the students and presented inworld.

This study lasted for two months and followed the HCI course programme. The participants formed eight groups (groups of 3-4 students) and each group had to design user interfaces for different interactive systems. The overall educational objective was to teach the students all the phases of the User-Centric Design (UCD) process utilizing a Virtual World. To undertake the learning tasks and activities, the same virtual world environment and the respective in-world collaboration tools and techniques (i.e., live text and voice chatting, forums for comments, etc.) were employed just like the first laboratory study described in 
previous sections. Most of the students did not have any previous experience with such a VW. Thus, a series of introductory tutorials on virtual worlds like Second Life, OpenSim, etc. were conducted (i.e., how to configure an avatar, how to create objects, etc.).

After the introductory courses, the students were assigned to design and develop interfaces for several interactive systems (e.g., Realtor's Agency, Online Game Shop, University's Management System, Smart Home Management System, etc.) utilizing the VW environment. The interface design of each system was based on the Logical User-Centered Interactive Design (LUCID) methodology. ${ }^{26}$ Some indicative tasks the students had to undertake throughout the design/development cycle were: i) literature review on similar systems, ii) determine the typical users of the system, iii) analyse the interface's design, following the Hierarchical Task Analysis (HTA) methodology, iv) design and develop the system's interface prototypes (Image 4), v) design users' navigation model.

During the two-month project, the students participated in a weekly problembased learning activity through the VW. All students and the instructors created their avatars and all communication during the PBL activity was done through the VW environment. In each session, the students were initially given a problem. Then they had to discuss it using in-world chat tools and wrote notes such as, what information was known, what information was needed and they had to specify an action plan for working on the problem. Furthermore, the students engaged in an independent study on their learning issues, such as digital libraries and encyclopaedias and resource people (i.e., other avatars in the VW). Then, students shared and evaluated the resources they've gathered for the various learning issues and reviewed what they had learned from working on the problem. Throughout the PBL session, the instructors' avatars were acting as a facilitator and mentor; they asked the students to consider issues like, "what is it that you don't know?" or "where can you find that information?" or "what do you think should be done next?". The facilitators didn't provide clues to any of these questions, but rather, prompted the students to consider "next steps" and processes along the way, thus engaging them in a typical constructivist learning situation. Table 2 describes how typical PBL activities (according to $\left(\right.$ Barrett, 2005) ${ }^{27}$ ) were implemented in this study.

The main positive aspect was that the vast majority of the students felt that the VW provided the means for a fruitful collaboration. Students claimed that the environment functioned as a shared space where processes such as discussion and exchange of ideas, creation of new ideas and brainstorming were promoted in an effective and efficient way. For some of the students participation in this study provided a new way of communication and collaboration especially for those that didn't have similar experiences with others VWs before. Some of the students noted that the VW provided the opportunity for them to "prove their communication skills". Some of their comments are very expressive: 
- " "The VW helped us to share ideas for the design process".

- "I found the environment very useful while testing the interfaces we created".

- "Comments from teammates facilitated the learning process. Comment listener and chatting were the tools that helped me the most".

- "The VW helped us in the process of work allocation".

- "It helped me to be more patient with the other members of the team"

Table 2 - Implementation of typical PBL activities in the VW - adapted from (Barrett, 2005)

\section{Typical PBL activities}

1) Students are presented with a problem "Design interactive interfaces for the system of your choice according to the main user tasks you have identified"

2) Students discuss the problem in small groups.

They clarify the facts of the case.

They define what the problem is.

They brainstorm ideas based on the prior knowledge.

They identify what they need to learn to work on the problem, what they do not know (learning issues).

They reason through the problem and they specify an action plan for working on the problem

3) Students engage in independent study on their learning issues outside the tutorial. This can include: library, databases, the web, resource people and observations

4) They come back to the PBL tutorial(s) sharing information, peer teaching and working together on the problem
The "Resource" object was utilized to access external Web resources.

The "Comment Recorder" is used to record user messages

The Annotation tool is used to make notes while studying the learning issues respectively

The Chat tool was used for synchronous communication

The "Annotation" tool was used to share the findings of each student

The "InterfaceElement" object was used as a shared interactive canvas thus helping them to work together

5) They present their solution to the problem

6) They review what they have learned from working on the problem.

Participants engage in self, peer and tutor review of the PBL process and reflect on each person's contribution to that process
The "InterfaceElement" object was used in order to present their solution

Chat tool was used

Students were "flying" from island to island to see the work of their peers 
They also pointed out the affordance of persistence of the VW, which was a highly motivating factor to go on for a period of two months. Additionally they emphasized the ability to see the work and the progress that other groups had achieved. Such kind of transparency was unprecedented for the most of the students, which led to a greater engagement and positive feelings despite the several technical problems.

The main problems identified were very much alike as the ones found in the first study. There was a strong focus on handling technical problems that aroused. In addition students stated that they would need more 2D functionalities such as the possibility to embed and share applications from their desktop environment to the VW, to co-edit documents etc. Another problem that was common between the two empirical studies was the issue of familiarity with the environment. The majority of the students did not have previous experiences with such VWs, while some of them claimed that VW would have to be more "authentic" in terms of representational fidelity.

\section{Discussion}

The results and experiences of the user studies indicate that VWs have a considerable potential as constructivist learning environments, provided that learning interventions are designed as authentic problems, students are engaged in co-creation activities, and related tools and provided inside the VW. On the other hand, they still lack the usability and robustness of more traditional computer supported approaches, such as the Web, and a lot of time and effort has to be invested to properly prepare and support the learning environment throughout the courses.

A notable advantage of the use of the VW compared to other technologymediated approaches to PBL lies in the awareness and integration. The group progress was visible to all, so both the tutors and the groups could be aware of the activities that took place, observe and comment on the documents and solutions that were proposed. This integrated environment allowed remote users to collaboratively construct solutions and communicate in real-time using voice or text chat. In the second case they could also record their discussion for later use. Furthermore, the VW and the tools created for the course offered various collaboration capabilities that allowed the group to work on their solution in parallel and exchange opinions and ideas through messages, drawings and sketches. Finally, the creative freedom offered by the VW in the sense that students could modify their appearance and construct and decorate their own collaborative space was highly engaging for most of them. These results comply with the claims that VWs have significant potential as constructivist learning environments. Students were engaged in the medium and, despite the difficulties, managed to complete their tasks in a highly collaborative and constructive process. 
On the other hand, the problems identified were sometimes critical and required interventions from the instructors. These findings suggest that VWs are still not mature enough as learning environments, and further research is needed to improve their usability and effectiveness. Inexperienced users find it difficult to navigate and manipulate elements in 3D and the large number of available commands and parameters needed to make full use of the environment made the user interface quite complicated. Therefore, in-world support by experienced users should be available during the learning activities in order to assist new users to overcome such difficulties. Furthermore, PBL activities are based on student collaboration and there was a notable lack of collaborative work tools in the VW. Elements such as shared documents and sketchboards would be useful during these activities. Ideally, as some students suggested, these tools should be interfaced with well known desktop applications that they are familiar with, such as word processing and image processing programs. Finally, VWs are also lacking tools and metaphors for organizational coordination and awareness, e.g. means to visualize work progress, task dependencies, user roles and assignments. To overcome these issues, the design of VWs as constructivist learning environments has to take into account CSCW methods and practices in combination to intuitive metaphors for the $3 \mathrm{D}$ user interface.

\section{Notes}

${ }^{1}$ John Pearson, 'Investigating ICT Using Problem-Based Learning in Face-to-face and Online Learning Environments'. Computers \& Education 47 (2006): 56-73.

2 John R. Savery and Thomas M. Duffy, 'Problem Based Learning: an Instructional Model and its Constructivist Framework', in B.G. Wilson, ed., Designing constructivist learning environments, (Englewood Cliffs, 1995), 135-148.

${ }^{3}$ Cindy E. Hmelo-Silver, 'Problem-Based Learning: What and How Do Students Learn?', Educational Psychology Review 16 (2004): 235-266.

${ }^{4}$ Roisin Donnelly, 'Blended Problem-Based Learning for Teacher Education: Lessons Learnt', Learning, Media and Technology 31 (2006): 93-116.

5 Jancis K. Dennis, 'Problem-Based Learning in Online vs. Face-to-face', Education for Health 16 (2003): 198-209.

${ }^{6}$ Selcuk Ozdemir, The Effects of Individual and Collaborative Problem-Based Learning Using an Online Asynchronized Learning Tool on Critical Thinking Abilities, Academic Achievements, and Attitudes Toward Internet Use, Phd dissertation, (Ankara: Gazi University Graduate School of Educational Sciences, 2005).

${ }^{7}$ Simon Bignell and Vanessa Parson, 'Best practice in Virtual Worlds Teaching: A Guide to Using Problem-Based Learning in Second Life', viewed 7 November 2011, Online archive available at <http://previewpsych.org/BPD2.0.pdf> 
${ }^{8}$ Christos Bouras, Eri Giannaka and Thrasyvoulos Tsiatsos, 'Virtual Collaboration Spaces: the EVE Community', Proceedings of 2003 Symposium on Applications and the Internet (2003): 48-55.

9 Andrea De Lucia, Rite Francese, Ignazio Passero and Genoveffa Tortora, 'Development and Evaluation of a Virtual Campus on Second Life: The Case of SecondDMI', Computers \& Education 52 (2009): 220-233.

${ }^{10}$ Teresa Monahan, Gavin McArdle, and Michela Bertolotto, 'Virtual reality for Collaborative E-Learning', Computers \& Education 50 (2008): 1339-1353.

${ }^{11}$ Elaine Brown, Marie Gordon and Mike Hobbs, 'Second Life as a Holistic Learning Environment for Problem-Based Learning and Transferable Skills', in Proceedings of Researching Learning in Virtual Environments Conference, (2008): 39-48.

12 Judith Good, Katherine Howland and Liz Thackray, 'Problem-Based Learning Spanning Real and Virtual Words: a Case Study in Second Life', Alt-J: Research in Learning Technology 16 (2008): 163-172.

13 Nicholas Omale, Wei-Chen Hung, Lara Luetkehans and Jessamine CookePlagwitz, 'Learning in 3-D Multiuser Virtual Environments: Exploring the use of Unique 3-D Attributes for Online Problem-Based Learning', British Journal of Educational Technology 40 (2009): 480-495.

${ }^{14}$ Shailey Minocha and Ahmad Reeves, 'Design of Learning Spaces in 3D Virtual Worlds: an Empirical Investigation of Second Life', Learning, Media and Technology 35 (2010): 111-137.

${ }^{15}$ Alvaro Sánchez, Jose María Barreiro, Victor Maojo, 'Design of Virtual Reality Systems for Education: A Cognitive Approach', Education and Information Technologies 5 (2000): 345-362.

${ }^{16}$ Carina Girvan and Timothy Savage, 'Identifying an Appropriate Pedagogy for Virtual Worlds: A Communal Constructivism Case Study', Computers \& Education 55 (2010): 342-349.

${ }^{17}$ Bignell and Parson, 'Best Practice in Virtual Worlds teaching: A Guide to Using Problem-Based Learning in Second Life'.

${ }^{18}$ Brown, Gordon and Hobbs, 'Second Life as a Holistic Learning Environment for Problem-Based Learning and Transferable Skills'.

${ }^{19}$ Good, Howland and Thackray, 'Problem-Based Learning Spanning Real and Virtual Words: a Case Study in Second Life'.

${ }^{20}$ Omale et al, 'Learning in 3-D Multiuser Virtual Environments: Exploring the use of Unique 3-D Attributes for Online Problem-Based Learning'.

${ }^{21}$ Ioannis Vrellis, Nikiforos M. Papachristos, Joan Bellou, Nikolaos Avouris and Tassos A. Mikropoulos, 'Designing a Collaborative Learning Activity in Second Life An Exploratory Study in Physics', in Proceedings of the 10th IEEE International Conference on Advanced Learning Technologies, (2010). 
22 'Open Simulator Home Page', viewed 7 October 2011, $<$ http://www.opensimulator.org $>$

23 'FreeSwitch Home Page', viewed 7 October 2011, <http://www.freeswitch.org >

24 Susan R. Fussell, Robert E. Kraut and Jane Siegel, 'Coordination of Communication: Effects of Shared Visual Context on Collaborative Work', in Proceedings of the 2000 ACM conference on Computer supported cooperative work, ACM, (2000): 21-30.

${ }^{25}$ Thanasis Daradoumis, Alejandra Martinez-Mones and Fatos Xhafa, 'A Layered Framework for Evaluating On-line Collaborative Learning Interactions', International Journal of Human-Computer Studies 64 (2006): 622-635.

${ }^{26}$ Charles B. Kreitzberg, The LUCID Framework: An Introduction, (New Jersey: Cognetics Corporation, 2000).

${ }^{27}$ Terry Barrett, Iain Mac Labhrainn and Helen Fallon, eds., Handbook of Enquiry and Problem-based Learning. (Galway: AISHE and CELT, 2005).

\section{Bibliography}

Barrett, Terry, Mac Labhrainn, Iain and Fallon, Helen, eds., Handbook of Enquiry and Problem-based Learning. Galway: AISHE and CELT, 2005.

Bignell, Simon and Parson, Vanessa. 'Best practice in Virtual Worlds teaching: A Guide to Using Problem-Based Learning in Second Life', Online archive available at http://previewpsych.org/BPD2.0.pdf [accessed Jan. 2011]

Bouras, Christos, Giannaka, Eri and Tsiatsos, Thrasyvoulos. 'Virtual Collaboration Spaces: the EVE Community'. In Proceedings of 2003 Symposium on Applications and the Internet, 48-55, 2003.

Brown, Elaine, Gordon, Marie and Hobbs, Mike. 'Second Life as a Holistic Learning Environment for Problem-Based Learning and Transferable Skills'. In Proceedings of Researching Learning in Virtual Environments Conference, 39-48, 2008.

Daradoumis, Thanasis, Martinez-Mones, Alejandra and Xhafa, Fatos. 'A Layered Framework for Evaluating On-line Collaborative Learning Interactions'. International Journal of Human-Computer Studies 64 (2006): 622-635. 
De Lucia, Andrea, Francese, Rite, Passero, Ignazio and Tortora, Genoveffa. 'Development and Evaluation of a Virtual Campus on Second Life: The Case of SecondDMI'. Computers \& Education 52 (2009): 220-233.

Dennis, Jancis K. 'Problem-Based Learning in Online vs. Face-to-face'. Education for Health 16 (2003): 198-209.

Donnelly, Roisin. 'Blended Problem-Based Learning for Teacher Education: Lessons Learnt'. Learning, Media and Technology 31 (2006): 93-116.

Fussell, Susan R., Kraut Robert E. and Siegel, Jane. 'Coordination of Communication: Effects of Shared Visual Context on Collaborative Work'. In Proceedings of the 2000 ACM conference on Computer supported cooperative work, 21-30. ACM, 2000.

Girvan, Carina and Savage, Timothy. 'Identifying an Appropriate Pedagogy for Virtual Worlds: A Communal Constructivism Case Study'. Computers \& Education 55 (2010): 342-349.

Good, Judith, Howland, Katherine and Thackray, Liz. 'Problem-Based Learning Spanning Real and Virtual Words: a Case Study in Second Life'. Alt-J: Research in Learning Technology 16 (2008): 163-172.

Hmelo-Silver, Cindy E. 'Problem-Based Learning: What and How Do Students Learn?'. Educational Psychology Review 16 (2004): 235-266.

Kreitzberg, Charles B. The LUCID Framework: An Introduction. New Jersey: Cognetics Corporation, 2000.

Minocha, Shailey and Reeves, Ahmad. 'Design of Learning Spaces in 3D Virtual Worlds: an Empirical Investigation of Second Life'. Learning, Media and Technology 35 (2010): 111-137.

Monahan, Teresa, McArdle, Gavin and Bertolotto, Michela. 'Virtual Reality for Collaborative E-Learning'. Computers \& Education 50 (2008): 1339-1353.

Omale, Nicholas, Hung, Wei-Chen, Luetkehans, Lara and Cooke-Plagwitz, Jessamine. 'Learning in 3-D Multiuser Virtual Environments: Exploring the Use of Unique 3-D Attributes for Online Problem-Based Learning'. British Journal of Educational Technology 40 (2009): 480-495. 
Ozdemir, Selcuk. 'The Effects of Individual and Collaborative Problem-Based Learning using an Online Asynchronized Learning Tool on Critical Thinking Abilities, Academic Achievements, and Attitudes toward Internet Use', Phd dissertation, Ankara: Gazi University Graduate School of Educational Sciences, 2005 .

Pearson, John. 'Investigating ICT using Problem-Based Learning in Face-to-face and Online Learning Environments'. Computers \& Education 47 (2006): 56-73.

Sánchez, Alvaro, Barreiro, Jose María and Maojo, Victor. 'Design of Virtual Reality Systems for Education: A Cognitive Approach'. Education and Information Technologies, 5 (2000): 345-362.

Savery, John R. and Duffy, Thomas M. 'Problem Based Learning: an Instructional Model and its Constructivist Framework'. In Designing constructivist learning environments, edited by B.G. Wilson, 135-148, Englewood Cliffs, 1995.

Vrellis, Ioannis, Papachristos, Nikiforos M., Bellou, Joan, Avouris, Nikolaos and Mikropoulos, Tassos A. 'Designing a Collaborative Learning Activity in Second Life: An Exploratory Study in Physics'. In Proceedings of the 10th IEEE International Conference on Advanced Learning Technologies, 2010.

Spyros Vosinakis is a Lecturer at the Department of Product and Systems Design Engineering, University of the Aegean, Greece. His research interests include: Virtual Reality, Intelligent Virtual Agents, Collaborative Virtual Environments, Adaptation and Personalization in 3D Environments.

Panayiotis Koutsabasis is a Lecturer at the Department of Product and Systems Design Engineering, University of the Aegean, Greece. His research interests include: design and evaluation methods for HCI, natural HCI, intelligent and personalized interaction, User Experience studies and systems thinking in Interaction Design.

Panagiotis Zaharias is a Visiting Academic at the Department of Computer Science, University of Cyprus. His research interests include: E-learning design, Usability and User experience evaluation methods, Virtual worlds in education and training, Serious games and MMOGs. 
Marios Belk is a Ph.D. Student and Research Associate at the Department of Computer Science, University of Cyprus. His research interests include: Web Adaptation and Personalization Environments and Systems, Database Systems, Ontologies, Internet Technologies and the Semantic Web. 